\title{
Efeitos da densidade de plantio e da adição de nutrientes na produção de mudas de gramas halófitas em recipientes ${ }^{1}$
}

\author{
Adriana Martins Guedes de Azevedo ${ }^{2 *}$, César Serra Bonifácio Costa ${ }^{3}$, \\ Carlos Eduardo da Silva Leal ${ }^{4}$, Juliano César Marangoni ${ }^{5}$
}

10.1590/0034-737X201663010011

\section{RESUMO}

As gramas halófitas Spartina alterniflora e Spartina densiflora são espécies bioengenheiras, que podem ser utilizadas para mitigação de áreas degradadas de marismas e manguezais, para o controle da erosão costeira e para estabilização de dragado depositado em regiões estuarinas e costeiras. O objetivo deste trabalho foi avaliar os efeitos da densidade de plantio e da adubação com nitrogênio $(\mathrm{N})$ e fósforo $(\mathrm{P})$ sobre mudas de propagação vegetativa destas duas espécies, crescendo em bandejas $\left(0,15 \mathrm{~m}^{2} ; 7500 \mathrm{~cm}^{3}\right)$. Para isto, foram realizados em estufa agrícola, não climatizada, dois experimentos nos anos de 2009 e 2011. Em 2009, apenas S. alterniflora foi cultivada, em duas densidades de plantios (133 e 400 mudas $\mathrm{m}^{-2}$ ) e com dois níveis de adição de nutrientes (sem adubação e com adição total de 50,8 gN $\mathrm{m}^{-2}$ e $16 \mathrm{gP} \mathrm{m}^{-2}$ ). Em 2011, bandejas com 80 mudas $\mathrm{m}^{-2}$ de ambas as espécies foram adubadas com razões $2 \mathrm{~N}: 1 \mathrm{P}, 6 \mathrm{~N}: 1 \mathrm{P}$, 10N:1P e 14N:1P (adição total de $115 \mathrm{gN} \mathrm{m}^{-2}$ ). A adubação com NP estimulou a formação foliar e, em recipientes fertilizados, o número médio de hastes de $S$. alterniflora, após 80 dias, foi o dobro do observado nos recipientescontrole. Entretanto, densidades iniciais de 400 ou mais hastes $\mathrm{m}^{-2}$ nas bandejas resultaram em alongamento vertical excessivo das hastes de S. alterniflora (cerca de $100 \mathrm{~cm}$ de altura), o que prejudica o manuseio e o plantio. A adubação com razão $2 \mathrm{~N}$ :1P resultou em melhor perfilhamento de ambas as espécies.

Palavras-chave: Spartina spp., fertilização, perfilhamento, propagação vegetativa.

\section{ABSTRACT \\ The effects of planting density and nutrient addition on container-grown vegetative propagules of halophytic grasses}

The halophytic grasses Spartina alterniflora and Spartina densiflora are bioengineer species, which can be utilized on the mitigation of disturbed areas of salt marshes and mangroves, for coastal erosion control and to stabilize dredged material disposed in estuarine and coastal areas. The effects of planting density and fertilization with nitrogen $(\mathrm{N})$ and phosphorus $(\mathrm{P})$ on container-grown $\left(0.15 \mathrm{~m} 2 ; 7500 \mathrm{~cm}^{3}\right)$ vegetative propagules of both species were evaluated under greenhouse conditions, through two experiments during the years 2009 and 2011. In 2009, only S. alterniflora was cultivated within 2 planting densities (133 and 400 propagules $\mathrm{m}^{-2}$ ) and 2 levels of fertilization (without addition and with total addition of $50.8 \mathrm{gN} \mathrm{m}^{-2}$ and $16 \mathrm{gP} \mathrm{m}^{-2}$ ). In 2011, containers with 80 propagules $\mathrm{m}^{-2}$ of both species received $\mathrm{N}$ and $\mathrm{P}$ fertilization rates of $2 \mathrm{~N}: 1 \mathrm{P}, 6 \mathrm{~N}: 1 \mathrm{P}, 10 \mathrm{~N}: 1 \mathrm{P}$ and $14 \mathrm{~N}: 1 \mathrm{P}$ (for a total addition of $115 \mathrm{gN} \mathrm{m}^{-2}$ ). NP fertilization stimulated leaf formation and, in fertilized containers, the average number of S. alterniflora tillers was twice larger than

\footnotetext{
Submetido em 21/11/2013 e aprovado em 10/09/2015

${ }^{1}$ Este trabalho é parte da Tese de Doutorado da primeira autora.

Universidade Federal do Rio Grande, Instituto de Oceanografia, Rio Grande, Rio Grande do Sul, Brasil. amg.azv@ gmail.com

${ }^{3}$ Universidade Federal do Rio Grande, Laboratório Biotecnologia de Halófitas, Instituto de Oceanografia, Rio Grande, Rio Grande do Sul, Brasil. docosta@ furg.br

${ }^{4}$ Universidade Federal do Rio Grande, Laboratório Biotecnologia de Halófitas, Instituto de Oceanografia, Rio Grande, Rio Grande do Sul, Brasil. cadu.leal@gmail.com

5 Universidade Federal do Rio Grande, Laboratório de Estatística Ambiental, Instituto de Matemática, Estatística e Física, Rio Grande, Rio Grande do Sul, Brasil. julianomarangoni@furg.br

*Autora para correspondência: amg.azv@gmail.com
}

Rev. Ceres, Viçosa, v. 63, n.1, p. 076-085, jan-fev, 2016 
control treatments. However, initial planting densities of 400 or more propagules $\mathrm{m}^{-2}$ resulted in excessive vertical growth of $S$. alterniflora tillers (about $100 \mathrm{~cm}$ height), which can result into plant losses during handling and transplantation. For both species, the $2 \mathrm{~N}$ : $1 \mathrm{P}$ fertilization rate resulted in higher tillering.

Key words: Spartina spp., fertilization, tillering, vegetative propagation.

\section{INTRODUÇÃO}

O plantio das gramas do gênero Spartina (Poaceae) tornou-se uma alternativa padrão para mitigação de áreas entremarés degradadas, bem como para o controle de erosão e a disposição de dragado em regiões estuarinas e costeiras (Broome et al., 1983; Chung, 2004; Mendonça \& Costa, 2008; Costa et al., 2012). Gramas deste gênero são tolerantes a altas salinidades (halófitas) e reconhecidas como espécies bioengenheiras, por suas capacidades de colonizar áreas desnudas alagadas periodicamente pelo mar, de reduzir a hidrodinâmica e de promover a deposição do sedimento com sua densa copa, além de fixar o sedimento depositado com seu sistema radicular (Chung, 2004, Mendonça \& Costa, 2008; Costa et al., 2012; Marangoni \& Costa, 2012). O recobrimento da zona entremarés por estas gramas e outras plantas herbáceas halófitas possibilita a formação de banhados alagados por água do mar, denominados de marismas ou salgados, tanto em estuários como em lagunas, baías e ilhas de barreira. Estes ambientes ocorrem em costas temperadas e subtropicais, mas também, ao longo dos trópicos, nas bordas dos bosques de mangue (Adam, 1990; Costa \& Davy, 1992; Costa et al., 2009). A legislação federal brasileira (Lei n ${ }^{\circ} 12.651$ 25/05/2012) define as marismas como áreas de proteção permanente (APP), vitais para proteção da costa contra a erosão marinha, para sustentação da biodiversidade e dos recursos pesqueiros pelo oferecimento de habitats, além de determinante dos ciclos biogeoquímicos locais por suas altas taxas de produção primária. Entretanto, as marismas têm sido degradadas com a ocupação humana da costa e, mais recentemente, pela elevação relativa do nível do mar, relacionada com o aquecimento global (Costa et al., 2009).

Mudas de gramas do gênero Spartina podem ser obtidas em casa de vegetação ou em canteiros abertos, a partir da germinação de sementes e de propágulos vegetativos (hastes com fragmentos de rizomas e raízes) coletados nas próprias áreas de marisma a serem recuperadas (Chung, 2004; Mendonça \& Costa, 2008; Costa, 2011a; 2011b). A produção de mudas em recipientes possibilita melhor controle da nutrição e proteção das raízes contra os danos mecânicos e a desidratação (Ristvey et al., 2007; Gomes et al., 2009), bem como menor custo de produção e manejo mais adequado no viveiro e no plantio (Costa, 2011a; 2011b). Para otimização da produção de mudas em recipientes, é necessária a avaliação da resposta de crescimento ao adensamento de indivíduos. Nas marismas, gramas Spartina crescem em densidades de até 5000 hastes $\mathrm{m}^{-2}$ e suas raízes tendem a se concentrar no sedimento superficial, por causa da anoxia em camadas mais profundas (Nieva et al., 2001; Costa et al., 2003; Daleo et al., 2008). Logo, estas plantas apresentam um grande potencial de produção de mudas em recipientes, mas poucos estudos avaliaram esta possibilidade fitotécnica (Deng et al., 2008; Costa et al., 2011a; 2011b) e faltam quantificações do efeito da densidade de plantio sobre a formação de perfilhos.

Gramas de marismas podem-se desenvolver em condições nutricionais muito variáveis. No ambiente natural, o nitrogênio $(\mathrm{N})$ tem sido considerado o principal elemento limitante do crescimento de plantas de Spartina (Mendelssohn, 1979; Levine et al., 1998; Emery et al., 2001; Rickey \& Anderson, 2004). Spartina alterniflora requer muito nitrogênio em comparação com o fósforo (P), e a razão N:P, no tecido, sob condições de mínima necessidade para o crescimento, é de 16N:1P (Smart \& Barko, 1980). Broome et al. (1983) demonstraram que a disponibilidade de $\mathrm{P}$ rapidamente se torna limitante para S. alterniflora, à medida que aumenta a quantidade de $\mathrm{N}$ adicionada a plantios. Adicionalmente, planos de lama entremarés, com solos saturados, hipóxicos, frequentemente apresentam razões N:P tão baixas quanto 2,5N:1P (Marangoni, 2001; Mendonça \& Costa, 2008). A disponibilidade de P no sedimento pode influenciar tanto a ocupação dos solos pelos rizomas como a formação de perfilhos por Spartina (Marangoni, 2001; Costa et al., 2003; Mendonça \& Costa, 2008). Em sistemas convencionais de horticultura, fertilizantes com baixa razão N:P são frequentemente utilizados para estimular o desenvolvimento de raízes-rizomas em detrimento das estruturas aéreas (Ristvey et al., 2007). Um dos desafios para a produção de mudas de Spartina em casas de vegetação é a definição das condições ótimas de aplicação conjunta destes dois macronutrientes, em recipientes.

Em áreas entremarés da costa brasileira, são encontradas duas espécies do gênero Spartina, sendo uma, a $S$. 
alterniflora Loisel, que apresenta formação espaçada de suas hastes ao longo dos rizomas e desenvolve-se amplamente na parte mais baixa do piso entremarés (Adam, 1990; Costa \& Davy, 1992; Marangoni \& Costa, 2012) e, outra, a $S$. densiflora Brong., que forma moitas, pela pequena distância entre os entrenós das hastes e domina pisos entremarés médios e altos (Costa et al., 2003; Peixoto \& Costa, 2004). Estas espécies podem também coexistir em planos arenolodosos em processo de colonização e vêm sendo utilizadas em projetos de fitorremediação na zona costeira (Mendonça \& Costa, 2008; Costa, 2011a; 2011b; Marangoni \& Costa, 2012). Este estudo visou a avaliar os efeitos da densidade de plantio e da adição de $\mathrm{N}$ e de $\mathrm{P}$ sobre a produção de perfilhos e o desenvolvimento de mudas das gramas S. alterniflora e S. densiflora, cultivadas em recipientes, no ambiente de casa de vegetação.

\section{MATERIAL E MÉTODOS}

Dois experimentos de produção de mudas de Spartina foram realizados, respectivamente, na primavera de 2009 e no inverno-primavera de 2011, em uma estufa agrícola não climatizada localizada no Museu Oceanográfico "Prof. Eliézer de Carvalho Rios" da Universidade Federal do Rio Grande (FURG). Mudas de S. alterniflora e de $S$. densiflora foram obtidas, por propagação vegetativa, de plantas coletadas na marisma da Ilha da Pólvora (Rio Grande, RS, $32^{\circ} 02^{\prime} 06^{\prime \prime}$ S e $52^{\circ} 05^{\prime} 55^{\prime \prime} \mathrm{W}$ ), sendo que plantas de S. densiflora foram obtidas apenas em 2011. As plantas coletadas foram separadas em mudas, com uma haste (colmo), com raízes e fragmentos de rizomas. Essas mudas foram acomodadas em bandejas plásticas $(30 \mathrm{~cm}$ x $50 \mathrm{~cm}$; $\left.0,15 \mathrm{~m}^{2}\right)$, com areia fina de praia e composto orgânico (3:1), formando um leito de $5 \mathrm{~cm}$ de espessura no fundo das bandejas, para enraizamento e formação de perfilhos (volume de solo de $7500 \mathrm{~cm}^{3}$ ), conforme metodologia descrita por Mendonça \& Costa (2008) e Costa (2011b). A análise da areia de praia indicou a seguinte composição: $\mathrm{pH}=7,2$; $\mathrm{N}=0,3 \mathrm{mg} \mathrm{kg}^{-1} ; \mathrm{P}=0,5 \mathrm{mg} \mathrm{kg}^{-1} ; \mathrm{e} \mathrm{K}=19,0 \mathrm{mg} \mathrm{kg}^{-1}$. O composto orgânico tipo "terra preta" utilizado, da marca Humosolo Vida, foi previamente peneirado em malha de 5 $\mathrm{mm}, \mathrm{e}$ apresentou a seguinte composição: $\mathrm{pH}=5,6 ; \mathrm{N}=$ $7,7 \mathrm{~g} \mathrm{~kg}^{-1} ; \mathrm{P}=3,7 \mathrm{~g} \mathrm{~kg}^{-1} ; \mathrm{e} \mathrm{K}=1,6 \mathrm{~g} \mathrm{~kg}^{-1}$.

As bandejas foram distribuídas aleatoriamente na estufa e irrigadas diariamente com água doce de torneira. A cada 15-20 dias as plantas foram borrifadas com água do mar, que forneceu a suplementação de sódio necessária para estas plantas C4 halófitas (Smart \& Barko, 1980), além de limitar o crescimento de briófitas no sedimento e o ataque de insetos. Dados diários de temperatura na estufa foram obtidos com termômetro de mercúrio de máximas e mínimas.

\section{Experimento de 2009 - Densidade de plantio e adubação com $N$ e $P$}

O primeiro experimento foi conduzido, ao longo de 80 dias entre outubro e dezembro de 2009, visando a otimizar a densidade de plantio e a avaliar o efeito da adubação com N e P na produção de perfilhos de S. alterniflora. Mudas foram cultivadas nas bandejas de $0,15 \mathrm{~m}^{2}$ em duas densidades de plantios (20 mudas $=133$ plantas $\mathrm{m}^{-2} \mathrm{e} 60$ mudas $=400$ mudas $\mathrm{m}^{-2}$ ) e dois níveis de adição de nutrientes (sem adubação e com adição total de $50,8 \mathrm{gN} \mathrm{m}^{-2} \mathrm{e}$ $\left.16 \mathrm{gP} \mathrm{m}^{-2}\right)$. O delineamento experimental utilizado foi inteiramente ao acaso, com duas repetições hierarquizadas ("nested") em cada nível de densidade e de adição de nutrientes. Os nutrientes utilizados foram do tipo granulado industrial, marca "Ourofertil"; P como superfosfato (19\% de $\mathrm{P}_{2} \mathrm{O}_{5}$ ) e $\mathrm{N}$ como sulfato de amônio [21\% de $\left(\mathrm{NH}_{4}\right)_{2} \mathrm{SO}_{4}$ ]. A adição dos nutrientes foi dividida em duas aplicações, realizadas no início e após 40 dias de cultivo. A quantidade total de $\mathrm{N}$ e $\mathrm{P}$ utilizada foi baseada nos trabalhos de Mendelssohn (1979), Buresh et al. (1980), Levine et al. (1998), Emery et al. (2001) e Rickey \& Anderson (2004) que estudaram a limitação destes nutrientes para espécies de Spartina. As mudas mortas nas primeiras duas semanas de cultivo foram repostas. Após 30 dias e ao final dos 80 dias de cultivo, foram realizadas medições do número total de hastes novas (perfilhos), da altura (comprimento da maior folha da haste, a partir da base do colmo;em $\mathrm{cm}$ ) e do número de folhas vivas de cada haste.

\section{Experimento de 2011 - adubação com diferentes relações $N: P$}

$\mathrm{O}$ efeito da adubação com diferentes razões $\mathrm{N}$ e $\mathrm{P}$ na produção de perfilhos e no desenvolvimento de mudas de $S$. alterniflora e $S$. densiflora, em casa de vegetação, foi avaliado por meio de um experimento, empregando-se a técnica de propagação citada anteriormente. Para cada espécie, foram estabelecidas 12 bandejas $\left(0,15 \mathrm{~m}^{2}\right.$ cada), com 12 mudas por bandeja (144 mudas por espécie). As mudas mortas nas primeiras duas semanas de cultivo foram repostas. Após 30 dias, as bandejas foram designadas aleatoriamente para quatro grupos de três bandejas, sendo que cada grupo recebeu, uma única vez, respectivamente, a adição de $58,19,12 \mathrm{e} 8,2 \mathrm{~g} \mathrm{~m}^{-2} \mathrm{de} \mathrm{P}$ (superfosfato) por bandeja. Foram adicionadas $115 \mathrm{~g} \mathrm{~m}^{-2} \mathrm{de} \mathrm{N}$ (sulfato de amônio) em cada bandeja, quantidade cerca de duas vezes maior do que a utilizada no experimento de 2009. O delineamento experimental utilizado foi inteiramente ao acaso, com três repetições hierarquizadas ("nested") em cada um dos quatro níveis de adição de nutrientes, compostos pelas razões $2 \mathrm{~N}: 1 \mathrm{P}, 6 \mathrm{~N}: 1 \mathrm{P}, 10 \mathrm{~N}: 1 \mathrm{P}$ e $14 \mathrm{~N}: 1 \mathrm{P}$. Os nutrientes utilizados foram do tipo granulado industrial, marca "Ourofertil". No dia da adubação, as 12 mudas 
estabelecidas em cada bandeja tiveram quantificadas as suas alturas $(\mathrm{cm})$ e os números de folhas vivas e de perfilhos. Ao final do experimento, após 120 dias de cultivo, foram medidas novamente as alturas e os números de folhas vivas, de folhas mortas e de perfilhos das mudas originais.

\section{Análises Estatísticas}

Os dados dos dois experimentos foram analisados por meio de Análises de Variância (Zar, 1999) em termos de produção média de perfilhos por bandeja e do desenvolvimento individual das mudas, em cada tratamento testado. A produção de perfilhos de $S$. alterniflora por bandeja, ao final dos 80 dias do experimento de 2009 , de adição de nutriente $(\mathrm{Nu})$, em bandejas com diferentes densidades (De) de mudas, foi comparada por meio de ANOVA bifatorial (com fator de interação Nu x De). Para as duas espécies, a produção de perfilhos, após 120 dias do experimento de 2011, com mudas cultivadas em diferentes razões N:P, foi comparada por meio de ANOVA unifatorial. Estas análises foram realizadas tanto para valores absolutos como para valores percentuais, em relação à densidade de mudas plantadas por bandeja. Foi necessária a transformação dos dados percentuais, por meio da função Raiz Quadrada, para atender os pré-requisitos de normalidade e homocedasticidade da ANOVA (Zar, 1999). O teste "a posteriori” de contraste da Mínima Diferença Significativa de Fisher ("LSD”) foi utilizado para detectar diferenças significativas entre médias, a 5\% (Zar, 1999).

Para a análise do desenvolvimento individual das mudas de ambas as espécies, as médias de altura das hastes, dos números de folhas vivas, de folhas mortas e de perfilhos por haste foram comparadas entre os tratamentos, por meio de ANOVAs, para dados iniciais (30 dias) e finais dos dois experimentos. No experimento de 2009, apenas a altura e o número de folhas vivas por haste foram quantificados. O desenho utilizado nas ANOVAs foi o hierárquico ("nested"), com as bandejas aninhadas dentro dos tratamentos (densidades e nutrientes). O modelo hierárquico das ANOVAs não incluiu um fator de interação $\mathrm{Nu} x$ De, por causa da inserção do fator de variabilidade entre bandejas-réplicas (FB), dentro de cada tratamento. A avaliação dos efeitos dos tratamentos no desenvolvimento final das mudas, para os experimentos de 2009 e 2011, foi realizada utilizando-se, respectivamente, apenas parâmetros biométricos das dez e seis maiores mudas de cada bandeja (máximo desempenho (Costa et al., 2003). Por causa da arquitetura clonal de crescimento de plantas de Spartina (Costa \& Seeliger, 1988; Costa et al., 2003; Deng et al., 2008), após várias semanas de desenvolvimento e perfilhamento, cada bandeja possuía brotos recentes, com poucos centímetros, hastes jovens em expansão vertical e hastes maduras com folhas totalmente expandidas. Adotando-se a medida do máximo desempenho médio alcançado em cada condição experimental, pode-se prevenir a distorção da inserção da medição de brotos e hastes jovens, na avaliação do potencial de desenvolvimento de uma planta clonal. Com este expediente, as variáveis finais de ambas as espécies alcançaram os pré-requisitos de normalidade e homocedasticidade da ANOVA (Zar, 1999). Alguns parâmetros biométricos iniciais (30 dias) do experimento de 2011 foram transformados antes das ANOVAs, sendo estes o número de perfilhos por haste de S. alterniflora transformados, utilizando-se a função raiz quadrada, e os números de folhas vivas e de perfilhos por haste de $S$. densiflora, transformados por meio das funções $\log 10(x)$ e $\log 10(x+1)$. O teste LSD foi utilizado para detectar diferenças significativas entre médias, a 5\% (Zar, 1999).

\section{RESULTADOS}

\section{Experimento de 2009}

O experimento de 2009 foi desenvolvido entre o início de outubro e o final de dezembro de 2009, sendo que as médias ( \pm erro padrão) das temperaturas mínimas noturna e máximas diurna foram, respectivamente, de $16,3 \pm 1,0^{\circ} \mathrm{C}$ e $28,2 \pm 1,9^{\circ} \mathrm{C}$.

A produção de perfilhos (hastes) de S. alterniflora, por bandeja com adubação, foi maior do que a das não adubadas $(\mathrm{FNu}=20,9 ; \mathrm{p}<0,01)$ e as bandejas cultivadas com alta densidade apresentaram o maior número final de hastes $(\mathrm{FDe}=108,1 ; \mathrm{p}<0,001 ;$ Figura 1A). Entretanto, quando analisado o desempenho percentual da produção de perfilhos, em relação à densidade inicial de plantio, ocorreu apenas um efeito significativo da adição de nutrientes $(\mathrm{FNu}=8,4 ; \mathrm{p}<0,05)$. Após 80 dias de cultivo, as bandejas adubadas, cultivadas com densidade baixa e com densidade alta, apresentaram percentuais de aumento do número de mudas por perfilhamento em torno de $200 \%$, estatisticamente semelhantes $(\mathrm{FDe}=2,4 ; \mathrm{p}=0,193$; Figura 1A).

As médias iniciais (após 30 dias de cultivo) da altura das hastes (média global $=27,4 \pm 0,7 ; \mathrm{n}=320$ ) e do número de folhas vivas $(2,6 \pm 0,2$ folhas por haste), das mudas de S. alterniflora, não apresentaram diferenças significativas $(p>0,05)$ entre as bandejas designadas para os diferentes tratamentos de densidade de plantio e de adição de nutrientes (Tabela 1). Ao final dos 80 dias de cultivo, independentemente da densidade de plantio, as mudas adubadas mostraram alturas médias maiores ( $\mathrm{p}<0,001)$. Este ganho em altura com a adubação foi marcadamente superior para mudas crescendo em alta densidade $(99,8 \pm$ $0,9 \mathrm{~cm})$, resultando em efeitos significativos do tratamento densidade $(\mathrm{p}<0,001)$ e da variabilidade entre bandejas (FB; $\mathrm{p}<0,001$ ) (Tabela 1). O número médio de folhas vivas 
por haste apresentou diferenças significativas apenas entre mudas adubadas e não adubadas em alta densidade (FB; p < 0,05) (Tabela 1).

\section{Experimento de 2011}

O experimento de 2011 foi desenvolvido entre meados do mês de julho e novembro de 2011, sendo as médias das temperaturas mínimas noturna e máximas diurna, respectivamente, $14,1 \pm 0,6^{\circ} \mathrm{Ce} 25,1 \pm 0,9^{\circ} \mathrm{C}$.

Após 120 dias de cultivo, a produção média de hastes por bandeja, das mudas de $S$. alterniflora, cultivadas na razão $2 \mathrm{~N}: 1 \mathrm{P}(31,0 \pm 1,9$ hastes por bandeja; $258 \pm 16 \%$ da densidade de plantio) foi maior do que as das demais razões N:P (FNu = 4,24; p < 0,05; Figura 1B). S. densiflora demonstrou uma formação de mudas por perfilhamento 2 -
3 vezes maior do que $S$. alterniflora, entretanto não ocorreram diferenças significativas na produção média de hastes entre razões $\mathrm{N}: \mathrm{P}(\mathrm{FNu}=0,69 ; \mathrm{p}=0,580$; Figura $1 \mathrm{C})$.

Após 30 dias de cultivo (logo, antes da adição de nutrientes), mudas de ambas as espécies não apresentaram diferenças significativas $(\mathrm{p}>0,05)$ na altura, no número de folhas vivas por haste e no número de perfilhos por haste, entre as bandejas designadas para diferentes razões N:P. S. alterniflora exibiu valores médios iniciais de altura das hastes, oscilando entre $26,6 \pm 2,6$ e 29,4 \pm 1,5 $\mathrm{cm}$, de número de folhas vivas por haste entre $1,6 \pm 0,1 \mathrm{e}$ $1,9 \pm 0,1$ e do número de perfilhos por haste entre $0,75 \pm$ 0,02 e $0,84 \pm 0,04$. Para $S$. densiflora, as alturas iniciais das hastes variaram entre $27,0 \pm 1,6$ e 30,6 $\pm 1,7 \mathrm{~cm}$, o número de folhas vivas por haste entre $0,41 \pm 0,02$ e $0,47 \pm 0,02$ e o
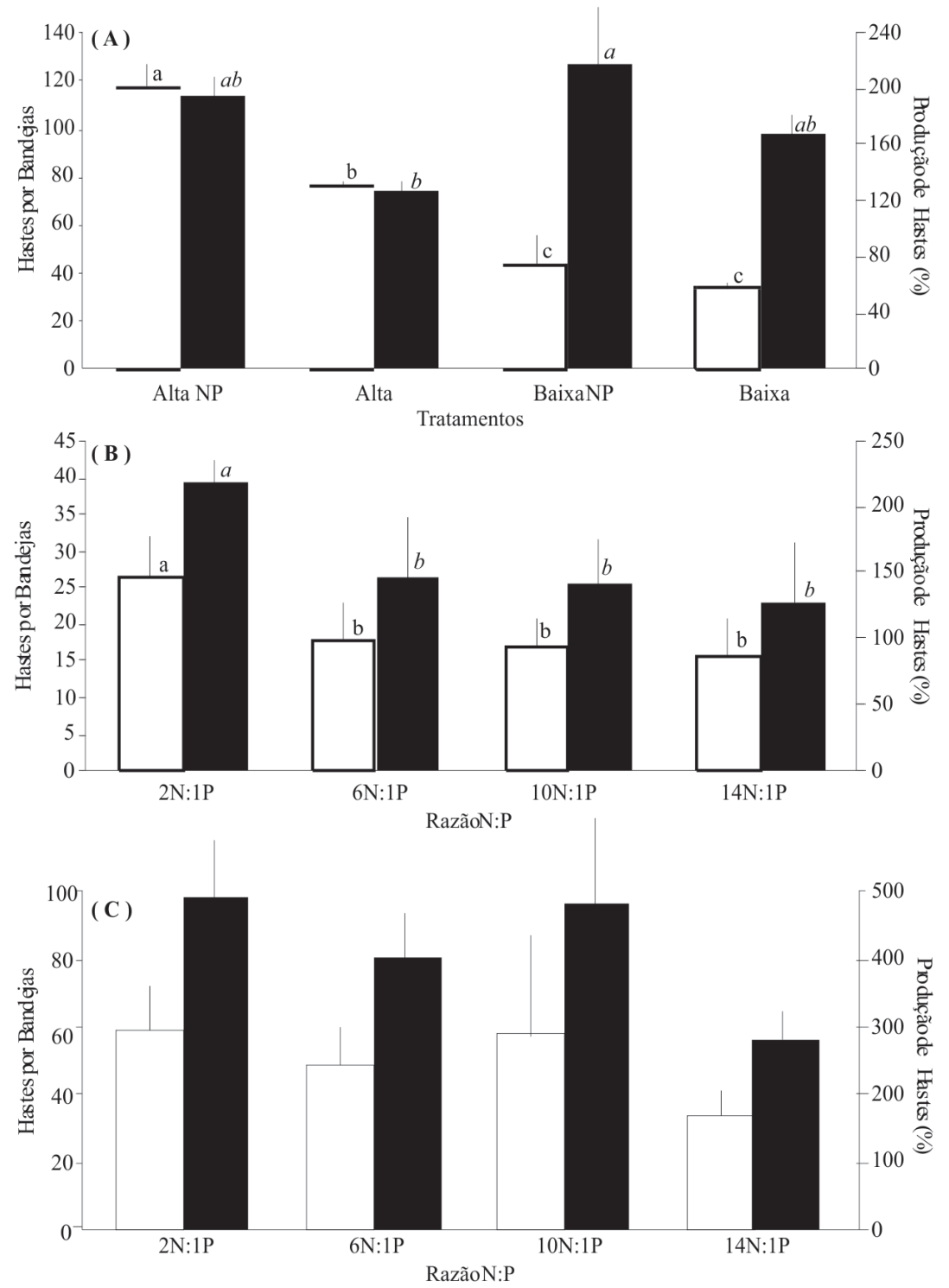

Figura 1: (A) Produção de hastes (perfilhos; média \pm erro padrão) de S. alterniflora, por bandeja com (NP) e sem adubação de nitrogênio e fósforo, em baixa e alta densidade inicial de mudas, após 80 dias de cultivo, em 2009. Produção de hastes (perfilhos) de S. alterniflora (B) e S. densiflora (C), por bandeja, em diferentes razões N:P, após 120 dias de cultivo, em 2011. Letras minúsculas diferentes entre barras de valores absolutos e percentuais (em itálico) indicam médias que diferem entre si pelo teste LSD ( $p<0,05)$. 
número de perfilhos por haste entre $0,07 \pm 0,02$ e $0,11 \pm$ 0,02 . Aos 30 dias, foram detectadas diferenças significativas, entre bandejas-réplicas de $S$. densiflora, quanto à altura das hastes $(\mathrm{FB}=2,27 ; \mathrm{p}<0,05)$ e ao número de perfilhos por haste $(F B=2,07 \mathrm{p}<0,05)$, principalmente pelas variações destes parâmetros entre bandejas do tratamento $6 \mathrm{~N}: 1 \mathrm{P}$.

Passados 120 dias de cultivo, ambas as espécies mostraram ótimo crescimento vegetativo. A razão N:P teve um efeito significativo sobre a altura e o número de perfilhos por haste de S. alterniflora, que aumentaram entre 14N:1P e 2N:1P (Figura 2A, D; Tabela 2). O número de folhas mortas por haste apresentou um padrão de resposta antagônico aos parâmetros acima citados, entre as razões N:P utilizadas (Figura 2C). As plantas submetidas à razão 2N:1P apresentaram uma média de folhas mortas por haste duas vezes menor do que das plantas crescendo nas demais razões N:P. O número de folhas vivas por haste, aos 120 dias, não apresentou diferença significativa entre níveis de nutrientes (Tabela 2; Figura 2B); logo, as plantas de $S$. alterniflora na razão $2 \mathrm{~N}: 1 \mathrm{P}$ mostraram uma menor renovação foliar do que as plantas submetidas à alta razão 14N:1P. Adicionalmente, também foi detectada uma variação significativa da altura e dos números de folhas vivas e de perfilhos por haste, entre bandejas dentro dos diferentes níveis de nutrientes, particularmente na razão $2 \mathrm{~N}: 1 \mathrm{P}$ (Tabela 2).

Ao final do experimento, as plantas de $S$. densiflora não tiveram nenhum parâmetro biométrico afetado pela razão N:P (Figura 2), mas o número de perfilhos variou significativamente, entre bandejas-réplicas, dentro dos níveis de nutrientes (Tabela 2).

\section{DISCUSSÃO}

\section{Densidade de plantio de $S$. alterniflora}

As mudas de $S$. alterniflora, crescendo em recipientes com $7500 \mathrm{~cm}^{3}$ de solo, atingiram, em 80 dias, densida- des médias finais de até 800 hastes $\mathrm{m}^{-2}$ (120 hastes por bandeja), valor maior do que as densidades naturais médias desta grama, observadas em marismas do estuário da

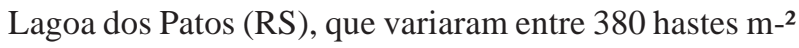
(Copertino et al., 1997) e 613 hastes $\mathrm{m}^{-2}$ (Costa et al., 2003). Sob adubação, mudas em menores densidades de plantio ( 20 mudas $=133$ hastes $\mathrm{m}^{-2}$ ) alocaram proporcionalmente mais recursos para formação de perfilhos do que as plantadas em alta densidade, mas atingiram apenas cerca de um terço da produção de hastes.

A adubação das plantas de $S$. alterniflora em altas densidades iniciais $\left(60\right.$ mudas $=400$ hastes $\left.\mathrm{m}^{-2}\right)$ resultou em marcado desenvolvimento da copa, em altura e número de folhas, desfavorável ao manejo. O manuseio, o transporte e o plantio destas plantas com cerca de um metro de altura exige uma demanda muito maior de tempo. Adicionalmente, plantas mais altas tendem a sofrer maior mortalidade, após o plantio na zona entremarés, por causa da ação de ondas e do maior empuxo, quando submersas (Castillo et al., 2005). A resposta de crescimento da copa de $S$. alterniflora em alta densidade também foi observada em populações naturais sujeitas a limitação de espaço e a alta disponibilidade de nitrogênio, sendo atribuída a uma resposta competitiva a redução da luz dentro da copa estabelecida (Levine et al., 1998; Emery et al., 2001; Pennings et al., 2002). A redução do tempo de cultivo dos perfilhos, formados em recipientes adubados, ou o plantio de mudas em densidades menores que 400 hastes $\mathrm{m}^{-2}$, pode permitir a otimização da produção de mudas com um tamanho mais apropriado ( $40-60 \mathrm{~cm}$ de altura), para manuseio e transplante para o ambiente natural.

\section{Razão N:P}

Os teores de nitrogênio $\left(50,8 \mathrm{a} 115 \mathrm{~g} \mathrm{~m}^{-2}\right)$ adicionados às bandejas, nos dois experimentos, mostraram um efeito estimulante ao desenvolvimento de mudas de $S$. alterniflora. Valores semelhantes foram empregados para

Tabela 1: Médias ( \pm erro padrão) iniciais (30 dias) e finais (80 dias) de altura e do número de folhas vivas das mudas de Spartina alterniflora, nos diferentes níveis de densidade de plantio (De) e de adição de nutrientes (Nu), no experimento de 2009. As médias finais dos parâmetros foram obtidas das 10 maiores plantas de cada bandeja de cultivo (máximo desempenho). Resultados dos testes de Fisher (F) para análises de variância também são apresentados para os tratamentos e variabilidade entre bandejas-réplicas (B)

\begin{tabular}{|c|c|c|c|c|c|c|c|}
\hline \multirow{2}{*}{$\begin{array}{l}\text { Tratamentos } \\
\text { D }\end{array}$} & \multirow[b]{2}{*}{$\mathrm{Nu}$} & \multirow[b]{2}{*}{$\mathbf{n}$} & \multicolumn{2}{|c|}{ Após 30 dias } & \multirow[b]{2}{*}{$\mathbf{n}$} & \multicolumn{2}{|c|}{ Após 80 dias } \\
\hline & & & Altura $(\mathrm{cm})$ & Folhas vivas & & Altura (cm) & Folhas vivas \\
\hline Alta & $\mathrm{np}$ & 120 & $30,9 \pm 1,1$ & $3,6 \pm 0,1$ & 20 & $99,8 \pm 0,9$ & $4,1 \pm 0,2 \mathrm{a}$ \\
\hline Alta & sem & 120 & $25,0 \pm 1,1$ & $3,0 \pm 0,1$ & 20 & $60,4 \pm 0,6$ & $3,2 \pm 0,2 b$ \\
\hline Baixa & $\mathrm{np}$ & 40 & $24,3 \pm 1,9$ & $3,4 \pm 0,1$ & 20 & $63,6 \pm 1,0$ & $3,7 \pm 0,2 \mathrm{ab}$ \\
\hline \multirow[t]{4}{*}{ Baixa } & sem & 40 & $27,0 \pm 1,9$ & $3,5 \pm 0,1$ & 20 & $61,9 \pm 1,6$ & $3,7 \pm 0,2 \mathrm{ab}$ \\
\hline & & $\mathrm{FNu}$ & $2,22 \mathrm{~ns}$ & $0,9 \mathrm{~ns}$ & $\mathrm{FNu}$ & $304,6 * * *$ & $0,3 \mathrm{~ns}$ \\
\hline & & FD & $1,04 \mathrm{~ns}$ & $2,29 \mathrm{~ns}$ & FD & $429,5 * * *$ & $7,21 * *$ \\
\hline & & $\mathrm{FB}$ & $2,08 \mathrm{~ns}$ & $1,94 \mathrm{~ns}$ & $\mathrm{FB}$ & $76,1 * * *$ & $2,69 *$ \\
\hline
\end{tabular}

$\overline{\mathrm{ns}}=\mathrm{p}>0,05$ (não significativo); $*=\mathrm{p}<0,05 ; * *=\mathrm{p}<0,01 ; * * * \mathrm{p}<0,001$. Letras minúsculas diferentes na vertical indicam médias que diferem entre si pelo teste LSD $(\mathrm{p}<0,05)$. 
adubação desta espécie no ambiente de marisma e também aumentaram o crescimento das plantas (Buresh et al., 1980; Broome et al., 1983; Levine et al., 1998; Emery et al., 2001; Pennings et al., 2002; Mendonça \& Costa, 2008). A forma granulada, utilizada dos fertilizantes, garantiu uma liberação lenta dos nutrientes ao longo do cultivo, confirmada pela boa qualidade fitossanitária final das plantas (folhas verde-escuro e vários perfilhos por haste).

A razão $2 \mathrm{~N}: 1 \mathrm{P}$ (maior quantidade de $\mathrm{P}$ ) estimulou a formação de perfilhos em recipientes das duas espécies de Spartina estudadas. A intensa formação de perfilhos por S. alterniflora, em solos com baixa razão N:P, foi anteriormente observada em marismas (Costa et al., 2003; Mendonça \& Costa, 2008). Broome et al. (1983) observa- ram que, frente a uma adubação de $22,4 \mathrm{gN} \mathrm{m}^{-2}$, em uma marisma no sudeste dos E.U.A., o aumento da adição de $\mathrm{P}$, de 1,2 para 9,9 $\mathrm{gP} \mathrm{m}^{-2}$ (redução da razão N:P de 18,6 para 2,2), resultou em um aumento de $46 \%$ do número de perfilhos de S. alterniflora. Cecato et al. (2008) também relatam a importância do fósforo no perfilhamento do capim-Mombaça (Panicum maximum), e que adubos fosfatados tiveram maior efeito sobre o número de perfilhos nesta grama, do que os adubos nitrogenados.

Além do maior perfilhamento, sob razão $2 \mathrm{~N}: 1 \mathrm{P}$ as plantas de $S$. alterniflora apresentaram redução da renovação foliar, evidenciada pelo menor número de folhas mortas. Gusewell (2004) observou que plantas perenes sob deficiência de $\mathrm{N}$ (adubação com baixas razões N:P)
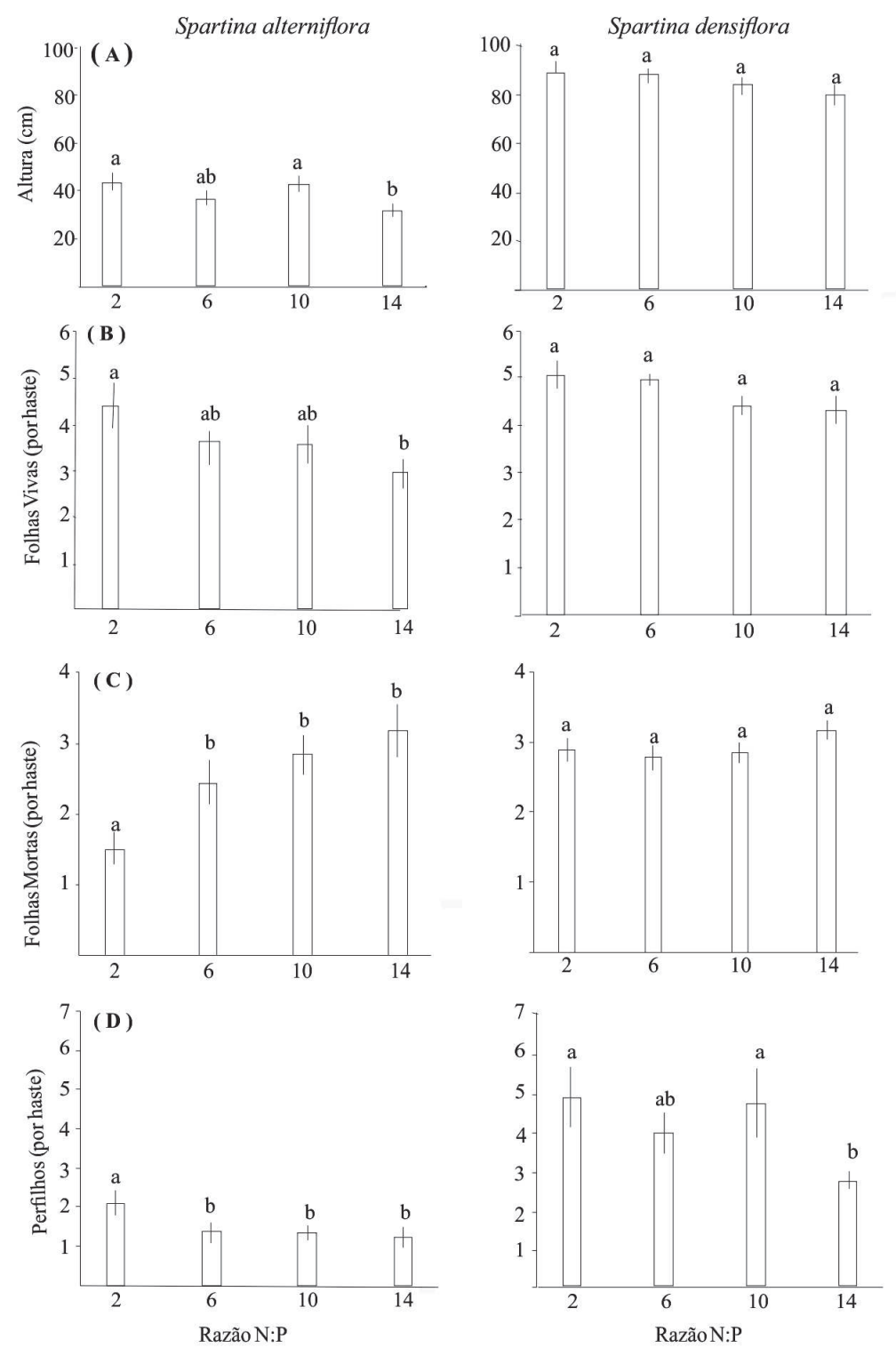

Figura 2: Máximo desempenho médio ( \pm erros padrões; 6 maiores plantas) da altura das hastes (a), do números de folhas vivas (b), de folhas mortas (c) e de perfilhos (d) produzidos por hastes após 120 dias de cultivo de Spartina alterniflora e Spartina densiflora, em diferentes razões N:P de adição de nutrientes. Letras minúsculas diferentes entre barras indicam médias que diferem entre si pelo teste LSD $(\mathrm{p}<0,05)$.

Rev. Ceres, Viçosa, v. 63, n.1, p. 076-085, jan-fev, 2016 
apresentaram menores taxas de senescência foliar do que plantas submetidas a deficiência de $\mathrm{P}$ (altas razões N:P). Esse autor explica esta resposta pela mobilização de $\mathrm{P}$ das folhas velhas para as folhas jovens, quando da deficiência deste elemento, resultando na inibição da fotossíntese e na aceleração da senescência das folhas velhas. Este fenômeno também foi observado em plantas de S. alterniflora, que apresentam teores foliares de $\mathrm{P}$, diretamente associados à quantidade de adubação com $\mathrm{P}$, mas teores foliares de $\mathrm{N}$ sem alteração ou mesmo menores frente à adubação com N (Broome et al., 1983). Adicionalmente, Smart (1994) sugeriu que o acúmulo de citocininas no tecido das plantas pode ser responsável por altas taxas de perfilhamento e por atraso da senescência das folhas maduras. Estes hormônios vegetais, sintetizados e transportados das raízes até os perfilhos, têm sua produção controlada ou regulada pelo suprimento de $\mathrm{N}$ ou de $\mathrm{P}$ (Gusewell, 2004). O papel destes hormônios no perfilhamento de plantas de $S$. alterniflora deveria ser futuramente analisado.

Exceto pela formação de perfilhos, todas as demais variáveis biométricas quantificadas de $S$. densiflora foram pouco influenciadas pela variação da razão $\mathrm{N}: \mathrm{P}$ de cultivo. Respostas contrastantes à adição de nutrientes por $S$. densiflora e $S$. alterniflora já haviam sido observadas anteriormente. Em um experimento com parcelas monoespecíficas de marismas na Argentina, Daleo et al. (2008) observaram após 16 meses de adubação com NPK (19:5:5) apenas um incremento de 60\% da densidade de hastes de $S$. densiflora, em relação a parcelas-controles (de 1632 para 2604 hastes $\mathrm{m}^{-2}$ ), enquanto S. alterniflora respondeu com um aumento da altura média da copa de $65 \%$ (de 60 para $99 \mathrm{~cm}$ ) e dobrou o número de hastes (de 133 para 277 hastes $\mathrm{m}^{-2}$ ). O denso perfilhamento é a principal estratégia utilizada por $S$. densiflora para ocupação espacial e exclusão de plantas competidoras, tendo sido observado em marismas populações com 900 a 5000 hastes $\mathrm{m}^{-2}$ (Nieva et al., 2001; Costa et al., 2003; Daleo et al., 2008; Costa \& Marangoni, 2012). Melhores eficiências nutricionais e capacidade de crescimento, em solos de marismas pobres em nutrientes, têm sido atribuídas às monocotiledôneas com pequena distância entre nós nos rizomas e que formam tufos de hastes, como $S$. densiflora. Estas habilidades seriam o resultado de uma maior razão biomassa rizomas-raízes:biomassa foliar e consequente capacidade de forragem por nutrientes (Levine et al., 1998; Emery et al., 2001; Costa et al., 2003; Daleo et al., 2008). A mistura de composto orgânico e areia mostrou ser adequada para o desenvolvimento de $S$. densiflora em recipientes. O ganho em perfilhamento pela adubação desta espécie deve ser considerado do ponto de vista econômico, antes da adoção desta prática.

A forma de crescimento em tufo de hastes, por $S$. densiflora, dificulta a separação das mudas para propagação vegetativa e parece ser a principal causa das diferenças no número de perfilhos produzidos por haste, entre bandejas-réplicas. O corte dos rizomas com distância entre nós reduzida, por vezes resulta no comprometimento de gemas basais (dos nós do rizoma). Adicionalmente, ao fragmentar os tufos de hastes das plantas-mãe, para preparar as mudas, há dificuldade para separar rizomas e raízes, o que resulta em biomassas subterrâneas desiguais e, consequentemente, desempenhos diferenciados em função de, possivelmente, diferentes números de gemas basais e, ou reservas nutricionais.

Tabela 2: Resultados das Análises de Variância de máximo desempenho (6 maiores plantas de cada bandeja de cultivo) da altura das hastes, do números de folhas vivas, de folhas mortas e de perfilhos produzidos por haste, após 120 dias de cultivo de Spartina alterniflora e Spartina densiflora em diferentes razões N:P de adição de nutrientes (Nu). O modelo de ANOVA utilizado considerou a variância do desenvolvimento das plantas relacionada com a bandeja de cultivo (B), aninhada em seu respectivo nível de adição de nutriente. Os graus de liberdade para a adição de nutrientes, variabilidade entre bandejas e variância residual foram, respectivamente, 3,8 e 58

\begin{tabular}{|c|c|c|c|c|c|c|c|}
\hline \multirow{2}{*}{ Variáveis } & \multicolumn{3}{|c|}{ Soma dos Quadrados } & \multirow[b]{2}{*}{ FNu } & \multicolumn{3}{|c|}{ Testes de Fisher } \\
\hline & $\mathrm{Nu}$ & B & Resíduo & & Sig & FB & Sig \\
\hline \multicolumn{8}{|c|}{ Spartina alterniflora } \\
\hline Altura $(\mathrm{cm})$ & 1591,61 & 2641,54 & 8392,35 & 3,4 & $*$ & 2,1 & $*$ \\
\hline Folhas vivas & 18,88 & 46,33 & 129,15 & 2,6 & ns & 2,4 & $*$ \\
\hline Perfilhos & 13,25 & 17,04 & 53,50 & 4,5 & $* *$ & 2,2 & $*$ \\
\hline Folhas mortas & 31,64 & 22,21 & 91,80 & 6,2 & $* * *$ & 1,6 & $\mathrm{~ns}$ \\
\hline \multicolumn{8}{|c|}{ Spartina densiflora } \\
\hline Altura (cm) & 768,94 & 3376,93 & 18660,39 & 0,8 & ns & 1,3 & ns \\
\hline Folhas vivas & 7,02 & 9,10 & 64,67 & 2,1 & ns & 1,0 & $\mathrm{~ns}$ \\
\hline Perfilhos & 51,11 & 197,67 & 383,00 & 2,7 & ns & 3,9 & $* * *$ \\
\hline Folhas mortas & 1,49 & 5,67 & 29,50 & 1,0 & $\mathrm{~ns}$ & 1,4 & ns \\
\hline
\end{tabular}

$\mathrm{ns}=\mathrm{p}>0,05$ (não significativo); $*=\mathrm{p}<0,05 ; * *=\mathrm{p}<0,01 ; * * * \mathrm{p}<0,001$. 


\section{CONCLUSÕES}

Plantas de S. alterniflora e de S. densiflora podem ser multiplicadas dentro de viveiro, por perfilhamento em recipientes de cultivo. A adubação com N (50,8 $\left.\mathrm{g} \mathrm{m}^{-2}\right)$ e P (16 gP m $\left.{ }^{-2}\right)$ estimulou a formação foliar e dobrou o número de hastes de $S$. alterniflora, após 80 dias de cultivo, no entanto, densidades iniciais de 400 ou mais hastes $\mathrm{m}^{-2}$, nos recipientes de cultivo, resultaram em alongamento vertical excessivo das hastes desta grama (cerca de $100 \mathrm{~cm}$ de altura), o que prejudica o manuseio e o plantio. A adubação com razão $2 \mathrm{~N}$ : $1 \mathrm{P}$ resulta em melhor perfilhamento de plantas de $S$. alterniflora e de $S$. densiflora.

\section{REFERÊNCIAS}

Adam P (1990) Saltmarsh Ecology. New York, Cambridge University Press. 461p.

Broome SW, Seneca ED \& Woodhouse Jr WW (1983) The effects of source, rate and placement of nitrogen and phosphorus fertilizers on growth of Spartina alterniflora transplants in North Carolina. Estuaries, 6:212-226.

Buresh RJ, Delaune RD \& Patrick Jr (1980) Nitrogen and phosphorus distribution utilization by Spartina alterniflora in a Louisiana gulf coast marsh. Estuaries, 3:111-121.

Castillo JM, Redondo S, Wharmby C, Figueroa ME, Luque T, Castellanos EM \& Davy AJ (2005) Environmental determination of shoot height in populations of the cordgrass spartina maritima. Estuaries, 28:761-766.

Cecato U, Skrobot VD, Fakir GR, Branco AF, Galbeiro S \& Gomes JAN (2008) Perfilhamento e características estruturais do capim-Mombaça, adubado com fontes de fósforo, em pastejo. Acta Scientiarum Animal Sciences, 30:1-7.

Chung CH (2004) Forty years of ecological engineering with Spartina plantations in China. Ecological Engineering, 27:4957.

Copertino M, Costa CSB \& Seeliger U (1997) Dinâmica populacional de Spartina alterniflora em pântanos salgados do estuário da Lagoa dos Patos, Rio Grande, RS. In: VIII Seminário Regional de Ecologia, São Carlos. Anais, UFSCar. p.295-312.

Costa CSB \& Seeliger U (1988) Demografia de folhas de Spartina ciliata Brong. em dunas e brejos costeiros. Revista Brasileira de Botânica, 11:85-94.

Costa CSB (2011a) Restoration of coastal salt marshes in Brazil using native salt marsh plants. In: Greipsson S (Ed.) Restoration Ecology. Sudbury, Jones and Bartlett Publishers. p.333-338.

Costa CSB (2011b) Projeto de recuperação de marismas. In: Tagliani PRA \& Asmus ML (Eds.) Manejo integrado do estuário da Lagoa dos Patos: uma experiência de gerenciamento costeiro no sul do Brasil. Rio Grande, Editora da FURG. p.179187

Costa CSB \& Davy AJ (1992) Coastal saltmarsh communities of Latin America. In: Seeliger U (Ed.) Coastal plant communities of Latin America. San Diego, Academic Press Inc. p.179-198.

Costa CSB, Marangoni JC \& Azevedo AMG (2003) Plant zonation in irregularly flooded salt marshes: relative importance of stress tolerance and biological interactions. Journal of Ecology, 91:951-965.
Costa CSB, Mendonça PTM \& Marangoni JC (2012) Criação de uma marisma em uma enseada estuarina no sul do Brasil utilizando sedimento dragado. In: VII Simpósio Brasileiro de Engenharia Ambiental (VII SBEA), Criciúma. Anais, Associação Brasileira de Engenharia Ambiental. p.960-969.

Costa CSB, Iribarne OO \& Farina JM (2009) Human impacts and threats to the conservation of South American salt marshes. In: Silliman BR, Grosholtz T \& Bertness MD (Eds.) Salt marshes under global siege. Berkeley, University of California Press. p.337-359.

Daleo P, Alberti J, Canepuccia A, Escapa M, Fanjul E, Silliman BR, Bertness MD \& Iribarne O (2008) Mycorrhizal fungi determine salt-marsh plant zonation depending on nutrient supply. Journal of Ecology, 96:43-437.

Deng Z, Shuqing A, Zhao C, Chen L, Zhou C, Zhi Y \& Li H (2008) Sediment burial stimulates the growth and propagule production of Spartina alterniflora Loisel. Estuarine, Coastal and Shelf Science, 76:818-826

Emery NC, Ewanchuk PJ \& Bertness MD (2001) Competition and salt-marsh plant zonation: stress tolerators may be dominant competitors. Ecology, 82:2471-2485.

Gomes-Neto A \& Costa CSB (2009) Survival and growth of the dominant salt marsh grass Spartina alterniflora in an oil industry saline wastewater. International Journal of Phytoremediation, 11:640-650.

Gusewell S (2004) N:P ratios in terrestrial plants: variation and functional significance. New Phytologist, 164:243-266.

Levine JM, Brewer JS \& Bertness MD (1998) Nutrients, competition and plant zonation in a New England salt marsh. Journal of Ecology, 86:285-292.

Marangoni JC (2001) Interação competitiva e habilidade colonizadora das plantas dominantes de uma marisma irregularmente inundada no estuário das Lagoa dos Patos (RS, Brasil). Tese de Doutorado. Rio Grande, Universidade Federal do Rio Grande. $169 \mathrm{p}$.

Marangoni JC \& Costa CSB (2012) Short- and long-term vegetative propagation of two Spartina species on a salt marsh in southern Brazil. Estuaries and Coasts, 35:763-773.

Mendelssohn IA (1979) The influence of nitrogen level, form, and application method on the growth response of Spartina alterniflora in North Carolina. Estuaries, 2:106-112.

Mendonça PTM \& Costa CSB (2008) Efeitos da disponibilidade de nitrogênio e fósforo no estabelecimento de Spartina alterniflora em um plano entremarés irregularmente alagado. Neotropical Biology and Conservation, 3:135-148.

Nieva FJJ, Diaz-Espejo A, Castellanos EM \& Figueiroa ME (2001) Field variability of invading populations of Spartina densiflora Brong. in different habitats of the Odiel marshes (SW Spain). Estuarine, Coastal and Shelf Science, 52:515-527.

Peixoto AR \& Costa CSB (2004) Produção primária líquida aérea de Spartina densiflora Brong. (Poacea) no estuário da Lagoa dos Patos, Rio Grande do Sul, Brasil. Iheríngia Série Botânica, 59:27-34.

Pennings SC, Stanton LE \& Brewer JS (2002) Nutrient effects on the composition of salt marsh plant communities along the southern Atlantic and Gulf coasts of the United States. Estuaries, 25:1164-1173.

Rickey M \& Anderson RC (2004) Effects of nitrogen addition on the invasive grass Phragmites australis and a native competitor Spartina pectinata. Journal of Applied Ecology, 41:888-896. 
Ristvey AG, Lea-Cox JD \& Ross DS (2007) Nitrogen and phosphorus uptake efficiency and partitioning of containergrown azálea during spring growth. Journal of American Society for Horticultural Science, 132:563-571.

Smart CM (1994) Gene expression during leaf senescence. New Phytologist, 126:419-448.
Smart RM \& Barko JW (1980) Nitrogen nutrition and salinity tolerance of Distichlis spicata and Spartina alterniflora. Ecology, 61:630-638.

Zar JH (1999) Biostatistical analysis. Nova Jersey, Prentice-Hall Inc. $718 \mathrm{p}$. 